\title{
Necesidades y dificultades de los/ las profesionales sanitarios/as de los servicios de prevención de riesgos laborales de Cataluña
}

\section{Needs and barriers of medical personnel in Catalonian occupational health services}

\author{
Hernán Vargas-Leguás ${ }^{1,3}$ (i) 0000-0002-5517-0360 \\ Eva Porcel Pérez ${ }^{2,3}$ (1) 0000-0002-9537-3592 \\ Imma Cortès-Franch 1,3,4,5 (i) 0000-0002-1244-9652 \\ 'Unitat de Salut Laboral de Barcelona. Servei de Salut i Treball. Agència de Salut Pública de Barcelona, \\ Barcelona, España. \\ ${ }^{2}$ Unitat de Salut Laboral de la Cataluña Central. Althaia Xarxa Assistencial Universitària de Manresa, \\ F.P. Manresa, Barcelona, España. \\ ${ }^{3}$ Xarxa de Unitats de Salut Laboral de Cataluña. Agència de Salut Pública de Cataluña, Barcelona, \\ España. \\ ${ }^{4}$ CIBER de Epidemiologia y Salud Pública (CIBERESP), Madrid, España. \\ ${ }^{5}$ Institut de Recerca de l'Hospital de la Santa Creu i Sant Pau (IIB-Sant Pau), Barcelona, España.
}

Fechas · Dates

Recibido: 2020.11.18

Aceptado: 2021.03.30

Publicado: 2021.04.15

\section{Correspondencia $\cdot$ Corresponding Author}

Hernán Vargas-Leguás

hvargas@aspb.cat 


\section{Resumen}

Objetivo: conocer las dificultades y necesidades del personal de medicina de los servicios de prevención de riesgos laborales de Cataluña para desarrollar sus funciones sanitarias descritas por la ley.

Métodos: estudio Delphi en tres rondas. La selección de participantes se realizó diferenciadamente para servicios de prevención propios y ajenos. La primera ronda incluyó dos preguntas abiertas: ¿Qué dificultades tiene para realizar las funciones sanitarias de su SPRL? y ¿Qué necesidades tiene para realizar las funciones sanitarias de su SPRL? En la segunda y tercera ronda las personas participantes puntuaron las categorías identificadas en la primera ronda según su importancia y relevancia. Se calcularon un índice de acuerdo interjueces (ICC) y un "índice de preocupación (IP)".

Resultados: la gestión de las personas trabajadoras con limitaciones y la vigilancia colectiva de la salud de baja calidad son las dificultades más importantes para los Servicios de Prevención Propios. Para los Ajenos, la falta de comunicación con otros organismos, la preeminencia de los criterios económicos y de rentabilidad asociado al alto volumen de trabajo son las más relevantes. La necesidad de establecer canales de comunicación con otras instituciones u organismos como la Atención Primaria, las Mutuas, el Instituto de Evaluaciones Médicas y las Unidades de Salud Laboral es común a ambos grupos.

Conclusiones: la diferente naturaleza de las actividades preventivas que realizan los servicios de prevención determina diferencias importantes en las dificultades expresadas, no así en las necesidades identificadas donde se encuentran puntos en común.

Palabras clave: Servicio de prevención de riesgos laborales; Salud Laboral; Personal sanitario de los servicios de prevención.

\section{Abstract}

Objective: to identify the difficulties and needs of medical professionals in occupational health services in Catalonia with respect to carrying out their functions, as prescribed by law.

Methods: Delphi study in three rounds. The selection of participants was carried out separately for in-house versus outsourced occupational health services. The first round included two open-ended questions: What difficulties do you encounter when performing your duties in your occupational health service? And, what are your needs in terms of being able to perform your duties in your occupational health service? In the second and third rounds, participants ranked the categories identified in the first round according to their importance and relevance. We calculated an inter-rater agreement index (ICC) and an "index of concern (IP)".

Results: Among in-house occupational health services, the most highly ranked difficulties were case management of workers with limitations and the low quality of medical surveillance systems. Among outsourced occupational health services, the most notable difficulties were the lack of communication with other organizations and the prioritization of economic and profitability criteria associated with the high work volume. For both types of services, there was a clear need to establish communication channels with other institutions or organizations such as primary care, mutual insurance companies, public medical evaluation units and public occupational health units. 
Conclusions: The different type of occupational health services reflects important differences in the types of difficulties encountered, but not in needs, where there are more points in common.

Keywords: Occupational Health Services; Occupational Health; Occupational Health Physicians.

\section{Introducción}

El trabajo es uno de los determinantes más importantes de la salud y el bienestar de las personas. La protección de las personas trabajadoras, la prevención de los riesgos en el trabajo y la promoción de la salud de la persona que trabaja es una parte esencial de la salud laboral y no solo la prevención de las lesiones ${ }^{(1)}$. Las intervenciones de salud laboral se llevan a cabo en España por tres instituciones: los servicios de prevención de riesgos laborales (SPRL) de las empresas, propios o ajenos; las Mutuas colaboradoras de la Seguridad Social, auto aseguradoras o el Instituto Nacional de la Seguridad Social y el Sistema Nacional de Salud(2). Según la ley, se entiende por servicio de prevención propio (SPP) el conjunto de medios humanos y materiales de la empresa necesarios para la realización de las actividades de prevención, y por servicio de prevención ajeno (SPA) el prestado por una entidad especializada que concierta con la empresa la realización de las actividades de prevención, el asesoramiento y apoyo que precise en función de los riesgos o ambas actuaciones conjuntamente ${ }^{(3)}$. Las actividades sanitarias de los servicios de prevención de riesgos laborales están descritas en el artículo 3 del Real Decreto 843/2011, por el cual se establecen los criterios básicos sobre la organización de los recursos para el desarrollo de la actividad sanitaria de los SPRL (Tabla 1).

En Cataluña, las Unidades de Salud Laboral (USL) son las encargadas de realizar las actuaciones de salud laboral en cada uno de sus ámbitos territoriales, para desarrollar las funciones que la normativa asigna al Departament de Salut de la Generalitat de Cataluña(4). Su distribución territorial coincide con la delimitación geográfica de las regiones sanitarias del Servicio Catalán de la Salud (CATSALUT).

Una de las actuaciones de las USL de Cataluña, de conformidad con la Ley de Salud Pública 18/2009(5), es dar apoyo a las empresas y servicios de prevención tanto propios como ajenos para que puedan cumplir sus actividades de salud laboral con la máxima calidad. Para materializar este apoyo, la Red de USL de Cataluña se ha propuesto desarrollar un plan de asesoramiento a los y las profesionales de Ios SPRL orientado a promover, supervisar y evaluar la calidad de sus actuaciones. Con el interés que este asesoramiento sea el más adaptado posible a las necesidades reales de los y las profesionales se propuso la realización de este estudio que tiene por objetivo conocer las dificultades y necesidades que tienen los/las profesionales sanitarios/as de los servicios de prevención de riesgos laborales de Cataluña para desarrollar sus funciones sanitarias descritas por el RD 843/2011(6). 


\section{Métodos}

Se desarrolló un estudio transversal cualitativo siguiendo la técnica Delphi( ${ }^{(7)}$ de tres rondas.

\section{Participantes}

La selección de las personas participantes del estudio se realizó en base a una muestra intencional o de conveniencia de forma separada según el tipo de servicio de prevención en el que trabajaban (SPP y SPA) y fueron propuestas por los responsables de las USL debido a su conocimiento del territorio y para asegurar que se trataba de informantes claves por la riqueza de información que podían aportar. Los criterios de selección fueron los siguientes: a) profesionales con al menos dos años de experiencia como personal sanitario de los SPRL, para poder aportar información relevante; b) presencia de servicios de prevención de todo el territorio de Cataluña, siguiendo la distribución territorial de las USL y en número proporcional al número de personas ocupadas en cada territorio, y c) inclusión de SPRL de empresas con diferentes actividades económicas. Se estableció un primer contacto telefónico entre los responsables de las USL y las personas participantes seleccionadas de su territorio para explicar brevemente el estudio y solicitar su autorización para compartir su dirección de correo electrónico. Posteriormente, el equipo investigador envió a todas las personas participantes un correo electrónico con un resumen del estudio, explicando los objetivos y los beneficios esperados de él. Los participantes respondieron dando su consentimiento para participar en el estudio. Se garantizó el tratamiento confidencial de la información, tanto de los datos de contacto como de las respuestas a las tres rondas.

\section{Recogida de información}

El proceso de consenso se realizó en tres rondas. Se envió un correo electrónico a las personas que aceptaron participar con el cuestionario y las instrucciones para responderlo. El primer cuestionario incluyó dos preguntas abiertas. Se solicitó expresamente que para responderlo se tuviera en cuenta la situación global del propio colectivo sanitario de los SPRL y especialmente las funciones descritas en el artículo 3 del RD 843/2011 de 17 de junio, por el que se establecen los criterios básicos sobre la organización de recursos para desarrollar la actividad sanitaria de los servicios de prevención ${ }^{(6)}$ (Tabla 1). Las preguntas del primer cuestionario fueron: ¿Qué dificultades tiene para realizar las funciones sanitarias de su SPRL? y ¿Qué necesidades tiene para realizar las funciones sanitarias de Su SPRL? Se utilizó la plataforma On-line Fluid Survey para realizar el cuestionario cuyo enlace se enviaba por correos electrónicos a las personas participantes. 
Tabla 1: Actividades sanitarias de los servicios de prevención. Artículo 3 del RD 843/2011.

\begin{tabular}{|c|c|}
\hline & Actividades descritas \\
\hline A & $\begin{array}{l}\text { Desarrollar todas aquellas funciones específicas recogidas en el apartado } 3 \text { del artículo } 37 \\
\text { del Reglamento de los Servicios de Prevención. }\end{array}$ \\
\hline B & $\begin{array}{l}\text { Estudiar, cuando se tenga conocimiento de ello, las enfermedades susceptibles de estar } \\
\text { relacionadas con el trabajo, a los solos efectos de poder identificar cualquier relación entre } \\
\text { las causas de enfermedad y los riesgos para la salud que puedan presentarse en los lugares } \\
\text { de trabajo. }\end{array}$ \\
\hline C & $\begin{array}{l}\text { Comunicar las enfermedades que podrían ser calificadas como profesionales, tal y como } \\
\text { establece el artículo } 5 \text { del Real Decreto 1299/2006, por el que se aprueba el cuadro de } \\
\text { enfermedades profesionales en el sistema de la Seguridad Social y se establecen criterios } \\
\text { para su notificación y registro, a través del organismo competente de cada comunidad } \\
\text { autónoma o de las ciudades con Estatuto de Autonomía. }\end{array}$ \\
\hline D & $\begin{array}{l}\text { Proporcionar la asistencia de primeros auxilios y la atención de urgencia a los } \\
\text { trabajadores que lo necesiten, en los casos de presencia física de los profesionales } \\
\text { sanitarios en el lugar de trabajo. }\end{array}$ \\
\hline$E$ & $\begin{array}{l}\text { Impulsar programas de promoción de la salud en el lugar de trabajo, en coordinación con el } \\
\text { Sistema Nacional de Salud. }\end{array}$ \\
\hline F & Desarrollar programas de formación, información e investigación en su ámbito de trabajo. \\
\hline G & $\begin{array}{l}\text { Efectuar sistemáticamente y de forma continua la vigilancia colectiva de la salud de los } \\
\text { trabajadores, en función de los riesgos a los que están expuestos, elaborando y disponiendo } \\
\text { de indicadores de dicha actividad. }\end{array}$ \\
\hline h & $\begin{array}{l}\text { Participar en las actuaciones no especificamente sanitarias que el servicio de prevención } \\
\text { realice en desarrollo de las funciones que tiene atribuidas conforme al apartado } 3 \text { del } \\
\text { artículo } 31 \text { de la Ley } 31 / 1995 \text {, de Prevención de Riesgos Laborales, a efectos de asegurar el } \\
\text { carácter interdisciplinario de dichas actuaciones. }\end{array}$ \\
\hline । & $\begin{array}{l}\text { Colaborar con el Sistema Nacional de Salud, tal y como establece el artículo } 38 \text { del } \\
\text { Reglamento de los Servicios de Prevención. }\end{array}$ \\
\hline J & $\begin{array}{l}\text { Colaborar con las autoridades sanitarias en las labores de vigilancia epidemiológica, } \\
\text { provisión y mantenimiento del Sistema de Información Sanitaria en Salud Laboral. }\end{array}$ \\
\hline K & $\begin{array}{l}\text { Participar en cualquier otra función que la autoridad sanitaria les atribuya en el marco de } \\
\text { la colaboración contemplada en los artículos } 38 \text { y } 39 \text { del Reglamento de los Servicios de } \\
\text { Prevención. }\end{array}$ \\
\hline
\end{tabular}

\section{Análisis}

Después de realizar un análisis de contenido de las respuestas recibidas en la primera ronda, el equipo investigador realizó un listado de categorías, que después de agruparlas y discutirlas, llevó a la elaboración de un listado con las dificultades y necesidades identificadas por las personas participantes. En la segunda ronda se envió un nuevo cuestionario que consistía en dos listados (las dificultades y las necesidades), con la instrucción de seleccionar las 10 más importantes de cada uno y darles un puntaje en una escala de 1 a 10 según su importancia y relevancia (1 para las menos importantes y 10 para las más importantes). Una vez recibidas las respuestas al segundo cuestionario se analizaron estos datos y se envió un tercer cuestionario (tercera ronda) con los resultados de las puntuaciones obtenidas en la segunda ronda para una última votación de prioridades. Todo el proceso se realizó mediante el correo electrónico. El análisis de los datos fue realizado por un equipo de tres personas investigadoras. Dos de ellas analizaron de forma 
independiente los datos de la primera ronda y elaboraron un listado común de las dificultades y necesidades identificadas. Cuando se produjo una discordancia, se presentó a una tercera persona para determinar las categorías definitivas. Los datos se analizaron por separado conservando siempre la diferencia entre SPP y SPA. Para demostrar el acuerdo interjueces, para la segunda y tercera ronda de puntuaciones se calculó el índice de correlación intraclase de acuerdo absoluto o ICC ${ }^{(8)}$ utilizando el programa STATA 15. Además, se calculó un "índice de preocupación (IP)" para unificar en un solo indicador el número de votos y el número de votantes [(número de votos * número de votantes) / número de participantes]. Este indicador permite tener en cuenta la extensión y la intensidad del problema con el objetivo de determinar el orden definitivo de los conceptos expresados. Se envió un informe con los resultados obtenidos a todas las personas que participaron en el estudio.

\section{Resultados}

Los resultados del estudio se presentan separadamente diferenciando los SPP y Ios SPA.

Respecto a los SPP, de las 25 personas seleccionadas que aceptaron participar en el estudio, 22 respondieron a la primera ronda, con una tasa de respuesta (TR) del $88 \%$. En la segunda ronda participaron 22 personas (TR 88\%) y la tercera ronda 19 participantes (TR 76\%). De las personas participantes en la primera ronda, 17 eran mujeres, 10 pertenecían a SPP de la administración, y de estas, 7 pertenecían al sector sanitario. En cuanto a la puntuación de las dificultades expresadas, el ICC en la segunda ronda fue de 0,70 (IC95\% 0,56-0,81) y el ICC de la tercera ronda fue de 0,80 (IC95\% 0,72-0,87). Respecto a las necesidades identificadas, el ICC de la segunda ronda fue de 0,79 (IC95\% 0,71-0,87) y el de la tercera ronda fue de 0,85 $(0,78-0,91)$.

De las 26 personas seleccionadas de los SPA que aceptaron participar, 23 respondieron a la primera ronda (TR 88\%), y 18 participantes a la segunda y tercera ronda (TR 70\%). De las personas participantes en la primera ronda, 18 eran mujeres y 10 trabajaban en SPA relacionados con las Mutuas Colaboradoras con la Seguridad Social. En cuanto a la puntuación de las dificultades expresadas por los participantes de los SPA, el ICC en la segunda ronda fue de 0,77 (IC95\% 0,68-0,85) y el de la tercera ronda fue de 0,89 (IC95\% 0,84-0,93). Respecto a las necesidades expresadas el ICC de la segunda ronda fue de 0,67 (IC95\% 0,54-0,78) y el de la tercera ronda fue de 0,87 (IC95\% 0,81-0,92).

En la Tabla 2 se observan las dificultades y necesidades que obtuvieron una puntuación de IP superior a 50 en la tercera ronda y que serán discutidas en el siguiente apartado de este artículo. Mientras que hubo poca diferencia en las puntuaciones de las principales dificultades de los SPP, se priorizaron dos necesidades claramente por encima de las otras (guías de buena práctica y protocolos de actuación, y grupos de trabajo multidisciplinarios). Los SPA, en cambio, priorizaron con claridad una dificultad y una necesidad relativas al mismo tema (falta de 
comunicación entre el personal sanitario implicado en la salud de las personas trabajadoras como principal dificultad y la consecuente necesidad de un sistema de comunicación entre dicho personal). En las tablas anexas 1, 2, 3, y 4 al final del artículo se pueden observar la totalidad del listado de dificultades y necesidades expresadas por los participantes, separadas por tipo de SPRL.

Tabla 2: Dificultades y necesidades de los/las profesionales sanitarios/as de los SPP y SPA para desarrollar sus actividades sanitarias. Resultados de la tercera ronda.

\begin{tabular}{|c|c|c|c|c|}
\hline Orden & Concepto & Puntos $^{a}$ & Participantes $^{\mathrm{b}}$ & IPc \\
\hline \multicolumn{5}{|c|}{ SERVICIOS DE PREVENCIÓN PROPIOS } \\
\hline \multicolumn{5}{|c|}{ Dificultades } \\
\hline 1 & $\begin{array}{l}\text { Dificultad para gestionar las personas que } \\
\text { son aptas con limitaciones (adaptación } \\
\text { del lugar de trabajo, cambios de lugar de } \\
\text { trabajo). }\end{array}$ & 116 & 13 & 79,4 \\
\hline 2 & $\begin{array}{l}\text { Hacer una vigilancia colectiva de la salud de } \\
\text { poca calidad. }\end{array}$ & 95 & 13 & 65,0 \\
\hline 3 & $\begin{array}{l}\text { Disparidad de criterios con las valoraciones } \\
\text { del ICAM. }\end{array}$ & 98 & 12 & 61,9 \\
\hline \multicolumn{5}{|c|}{ Necesidades } \\
\hline 1 & $\begin{array}{l}\text { Disponer de guías de buena práctica y } \\
\text { protocolos de vigilancia de la salud basados } \\
\text { en el consenso de los profesionales de la } \\
\text { salud laboral, adaptados a la realidad y con } \\
\text { evidencia científica. }\end{array}$ & 134 & 17 & 119,9 \\
\hline 2 & $\begin{array}{l}\text { Crear grupos de trabajo multidisciplinarios } \\
\text { (ICAM, Mutua, Unidad de salud laboral, SPP, } \\
\text { SPA) para compartir experiencias y buscar } \\
\text { soluciones. }\end{array}$ & 137 & 16 & 115,4 \\
\hline 3 & $\begin{array}{l}\text { Establecer colaboración con el personal } \\
\text { médico del ICAM. }\end{array}$ & 109 & 13 & 74,6 \\
\hline 4 & $\begin{array}{l}\text { Establecer una vía de comunicación con el } \\
\text { personal sanitario de los centros de atención } \\
\text { primaria. }\end{array}$ & 97 & 13 & 66,4 \\
\hline 5 & $\begin{array}{l}\text { Establecer criterios claros de limitaciones/ } \\
\text { restricciones según la patología y el lugar de } \\
\text { trabajo. }\end{array}$ & 88 & 11 & 50,9 \\
\hline \multicolumn{5}{|c|}{ SERVICIOS DE PREVENCIÓN AJENOS } \\
\hline \multicolumn{5}{|c|}{ Dificultades } \\
\hline 1 & $\begin{array}{l}\text { Falta de comunicación entre el personal } \\
\text { sanitario implicado en la salud de los } \\
\text { trabajadores y trabajadoras (SPRL, médico/ } \\
\text { ca de familia, especialista, Mutua, Unidad de } \\
\text { salud laboral, ...). }\end{array}$ & 154 & 17 & 145,4 \\
\hline 2 & $\begin{array}{l}\text { Que prevalezcan criterios económicos por } \\
\text { encima de los de calidad. }\end{array}$ & 112 & 14 & 87,1 \\
\hline 3 & Alto volumen de trabajo. & 110 & 13 & 79,4 \\
\hline
\end{tabular}




\begin{tabular}{|c|c|c|c|c|}
\hline Orden & Concepto & Puntos $^{a}$ & Participantes $^{\text {b }}$ & $\mathrm{IP}^{\mathrm{c}}$ \\
\hline 4 & $\begin{array}{l}\text { Tener que dedicar la mayor parte del tiempo } \\
\text { a la realización de exámenes de salud. }\end{array}$ & 91 & 13 & 65,7 \\
\hline 5 & $\begin{array}{l}\text { Presión de las empresas para obtener } \\
\text { resultados de acuerdo con sus intereses. }\end{array}$ & 86 & 11 & 52,6 \\
\hline \multicolumn{5}{|c|}{ Necesidades } \\
\hline 1 & $\begin{array}{l}\text { Establecer un sistema de comunicación } \\
\text { entre todo el personal sanitario implicado } \\
\text { en la atención de los trabajadores y } \\
\text { trabajadoras que permita tener acceso a } \\
\text { toda la información médica con la máxima } \\
\text { confidencialidad. }\end{array}$ & 151 & 17 & 142,6 \\
\hline 2 & $\begin{array}{l}\text { Establecer una legislación de prevención de } \\
\text { riesgos laborales más concreta i específica } \\
\text { que apueste por un mayor contenido en } \\
\text { medicina del trabajo y no solo en hacer } \\
\text { reconocimientos médicos. }\end{array}$ & 106 & 14 & 82,4 \\
\hline 3 & $\begin{array}{l}\text { Cambiar el modelo de organización de } \\
\text { los SPA, de manera que dependan de } \\
\text { organismos públicos con criterios y precios } \\
\text { comunes. }\end{array}$ & 107 & 13 & 77,3 \\
\hline 4 & Poder realizar interconsultas a especialistas. & 100 & 12 & 66,7 \\
\hline
\end{tabular}

\section{Discusión}

La gestión de las personas trabajadoras que son aptas con limitaciones en las empresas es la dificultad más importante identificada por los participantes de los SPP, lo cual se relaciona fundamentalmente con las adaptaciones o cambios de lugar de trabajo. Para el personal sanitario de los SPP la dificultad se presenta en su relación con los cargos intermedios y de dirección en las empresas para los cuales es difícil entender las tareas preventivas. Pese a que las actividades de prevención son un imperativo legal para las empresas ${ }^{(2)}$ y que las actividades sanitarias de los servicios de prevención están desarrolladas en un reglamento específico(5), observamos con estos resultados que las actuaciones de los SPP siguen siendo resistidas en las empresas. Los SPP al formar parte de las mismas empresas tienen información y contactos habituales con el personal y los cargos de dirección intermedios, sin embargo, las dificultades para realizar las adaptaciones o cambios en el lugar de trabajo de los y las trabajadoras que se reintegran después de una incapacidad temporal (IT), o de aquellos considerados como especialmente sensibles para los riesgos derivados del trabajo, son consideradas como elevadas por los participantes. Evidencia reciente demuestra la efectividad de estas intervenciones en las personas con trastornos musculo esqueléticos para disminuir el tiempo de las IT, reintegrarse más rápidamente al lugar de trabajo y mejorar el estatus funcional de las personas trabajadoras ${ }^{(0,10)}$. Esta evidencia es más limitada en cuanto a los trastornos de salud mental y está habitualmente enfocada a medir la efectividad de intervenciones individuales dirigidas a la persona trabajadora y 
no a su lugar de trabajo ${ }^{(11)}$. En coherencia con lo anterior, los SPP expresan como necesidad más relevante disponer de guías de buena praxis y protocolos de vigilancia de la salud consensuados y basados en la evidencia científica disponible, que sirvan como base para apoyar sus actuaciones en las empresas. También expresan la necesidad de establecer criterios de limitaciones o restricciones según la patología laboral y el lugar de trabajo.

La disparidad de criterios con el personal médico evaluador del Instituto Catalán de Evaluaciones Médicas (ICAM) que es quien realiza las funciones de inspección médica de las incapacidades en Cataluña, fue la tercera dificultad priorizada. Los profesionales participantes de los SPP expresan que sus valoraciones de las aptitudes para trabajar muchas veces son anuladas por parte del ICAM generando importantes dificultades para gestionar en las empresas a los y las trabajadoras que en la práctica no pueden desarrollar sus funciones, obligándoles a realizar un cambio del lugar de trabajo o adaptaciones no deseadas por las propias personas trabajadoras o por sus compañeros y compañeras de trabajo. La capacidad para trabajar no sólo depende de las características físicas o condición de salud de la persona que trabaja sino también de las características de su lugar de trabajo ${ }^{(12)}$. Esta última información que puede ser proporcionada por los SPP no debería ser olvidada por la autoridad inspectora. En este sentido, la colaboración con el personal médico del ICAM fue una de las necesidades más valoradas. Teniendo en cuenta esto, se hace necesario objetivar y comunicar los criterios para conceder o mantener una incapacidad laboral entre los diferentes actores que influyen en la salud de las personas trabajadoras, considerando tanto la patología, las características personales, así como la información del lugar de trabajo. Se han realizado esfuerzos en este sentido en el ámbito estatal ${ }^{(13,14)}$ que a la luz de lo expresado por los profesionales que participaron de los SPP aún son insuficientes. La creación de equipos de trabajo multidisciplinarios, expresada como una necesidad por los SPP, con participación de profesionales del ICAM, la atención primaria, las Mutuas y las USL puede ser el lugar adecuado para generar estos criterios comunes.

Según los profesionales que participaron de los SPP, la dificultad para realizar una vigilancia colectiva de la salud de calidad está relacionada con diferentes factores como el elevado volumen de trabajo, la utilización de herramientas informáticas no adecuadas y la dedicación prioritaria a la vigilancia individual de la salud de las personas trabajadoras, a través de los exámenes de salud. En nuestro medio, los exámenes de salud se realizan con insuficiente información clínica, escasa información de la exposición a los riesgos específicos en relación al puesto de trabajo, no son útiles para identificar a las personas trabajadoras especialmente sensibles, no permiten elaborar propuestas preventivas y no están exentos de causar perjuicios en la situación laboral de las y los trabajadores ${ }^{(15)}$. Las personas participantes lo explicaban así en la primera ronda:

\footnotetext{
"Se pasan muchos cuestionarios... pero los resultados obtenidos en las exploraciones y pruebas pocas veces se relacionan con los riesgos descritos de los puestos de trabajo y con los síntomas y signos que afectan a los trabajadores" (SPP29G); "Muchos esfuerzos en tiempo y dinero en lo individual y poca atención en la vigilancia colectiva" (SPP21B).
} 
Respecto a los SPA, la falta de comunicación entre el personal sanitario con las otras instituciones implicadas en la salud de las personas trabajadoras es reconocida por los participantes y por mucha distancia como la dificultad más importante para estos profesionales. La falta de comunicación se menciona principalmente con las Mutuas colaboradoras con la Seguridad Social, especialmente respecto al seguimiento de las personas en IT, pero también se mencionan dificultades en la comunicación con los profesionales de atención primaria del Sistema Público de Salud, los especialistas y las USL. Esta falta de comunicación puede influir en la efectividad de las acciones preventivas, en la investigación del origen laboral de las patologías, en la reincorporación y recuperación de los trabajadores y trabajadoras, según expresan las mismas personas participantes. La dificultad en la comunicación también ha sido informada en otros países de Europa poniendo énfasis en la utilidad de esta comunicación para facilitar la reintegración de las personas trabajadoras a sus lugares de trabajo ${ }^{(9,16-19)}$. Se ha descrito que los obstáculos para la comunicación se pueden organizar en tres apartados: i) organizacionales como la falta de datos de contacto compartidos, las restricciones de horarios; ii) interpersonales como la poca confianza entre los profesionales de unas y otras instituciones, la baja percepción de ayuda por parte de los médicos de atención primaria y el escaso interés en participar, y iii) barreras estructurales como los reglamentos de protección de datos o la privacidad de los informes mé$\operatorname{dicos}^{(19)}$. La necesidad de establecer un sistema de comunicación entre todo el personal sanitario involucrado en la salud de las personas trabajadoras se expresa con claridad por parte de los profesionales participantes de los SPA. Este sistema permitiría compartir la información sanitaria relevante y favorecería la continuidad asistencial de los procesos de cuidado y prevención de los problemas de salud relacionados con el trabajo. El requisito más importante es asegurar la confidencialidad de los datos derivados de la atención sanitaria. Recientemente, en Italia se ha propuesto como primer paso la elaboración de guías prácticas para estandarizar los procedimientos de comunicación entre los y las profesionales de atención primaria y de los servicios de prevención ${ }^{(18)}$.

Los participantes de los SPA destacan en segundo lugar la dificultad generada por la preeminencia de los criterios económicos por sobre los de calidad de la atención que prestan a los trabajadores y trabajadoras. Las exigencias para disminuir costos pueden, según las personas participantes, afectar la cantidad de recursos disponibles para la prevención y la periodicidad de los exámenes de salud. Los SPA están obligados por su naturaleza empresarial a ser rentables. Esta situación está muy relacionada con otra de las dificultades expresadas en el estudio: la injerencia de las empresas contratantes en las actividades de los SPA, que se expresa en la presión que reciben los profesionales para obtener determinados resultados de acuerdo con los intereses de las empresas, especialmente en relación con los dictámenes de aptitud para el trabajo y la identificación precoz de las patologías relacionadas con el trabajo. Los objetivos de rentabilidad de los SPA determinan una relación clientelar con las empresas contratantes que dificulta sus acciones preventivas y puede generar un conflicto moral importante en las personas que trabajan en estos servicios. Lograr un cierto equilibrio en la resolución de situacio- 
nes complejas que incluyen la gestión de estos conflictos es uno de los desafíos de futuro de los SPA siguiendo el modelo empresarial actual(20). Sin embargo, los participantes en este estudio expresan como una de las necesidades más relevantes cambiar el modelo organizativo de los SPA, proponiendo que dependan directamente de organismos públicos, con prestaciones y precios comunes, con el objetivo de eliminar la relación empresa-cliente, focalizando los esfuerzos en el trabajo preventivo. Por otro lado, este modelo propuesto favorecería la adquisición de criterios comunes y la comunicación con las otras instituciones involucradas en la salud de las personas trabajadoras.

Por las propias características de los SPA derivadas de ser un servicio externo a las empresas, la falta de información y contacto con los puestos de trabajo es mayor que para los SPP y su capacidad para influir con recomendaciones preventivas es mucho menor. Los participantes de los SPA también manifiestan las dificultades relacionadas con el alto volumen de trabajo y tener que dedicar la mayor parte del tiempo a los exámenes de salud individuales. La poca especificidad de las pruebas y analíticas realizadas rutinariamente unida a la falta de información específica del lugar de trabajo y sus riesgos, transforma estos exámenes de salud en chequeos médicos generales muy alejados de su finalidad preventiva (21). Estas dos dificultades (elevado volumen de trabajo y dedicación casi exclusiva a los exámenes de salud individuales) parecen estar relacionadas también con la priorización de criterios económicos por encima de la calidad, tal como algunas/ os de las/os participantes mencionaban en la primera ronda:

"A veces no tengo tiempo de hacer nada más que exámenes de salud, ni planificaciones, ni valoraciones del daño corporal, ni visitas a mis empresas, ni de mirar estudios epidemiológicos ni los otros aspectos que también son parte de mi trabajo pero que no son tan lucrativos (SPA24B)".

No sorprende que una de las necesidades más relevantes expresadas por los SPA sea mejorar la legislación en prevención de riesgos laborales para hacerla más concreta y específicamente dirigida a un mayor contenido de medicina del trabajo y actividades preventivas y no sólo a la realización de los exámenes de salud individuales.

Entre las fortalezas de este estudio se encuentran la alta participación de los informantes que participaron en las tres rondas del estudio y que, además, han demostrado un importante grado de acuerdo entre ellos. Lo que permite considerar que la validez externa del estudio es alta, teniendo en cuenta las limitaciones propias de los estudios cualitativos como este. La limitación más importante del estudio viene condicionada metodológicamente al tratarse de un estudio DELPHI que no permite lograr mayor profundidad en el análisis de las causas y determinantes de las categorías identificadas. Sin embargo, a partir del estudio se pueden visualizar líneas de investigación que permitan profundizar en los resultados obtenidos.

En conclusión, este estudio ha identificado las dificultades y necesidades más relevantes que tienen los profesionales de los SPP y SPA de Cataluña para desarrollar sus tareas sanitarias. La diferente naturaleza de las actividades preventivas 
que realizan estos servicios de prevención determina diferencias importantes en las dificultades expresadas, no así en las necesidades donde se encuentran puntos en común.

\section{Financiación}

Este trabajo no ha recibido financiación específica

\section{Conflicto de intereses}

Las personas autoras se declaran sin conflicto de intereses.

\section{Bibliografía}

1. Garcia AM, Benavides F, Ruiz-Frutos C. Salud Laboral. En: Ruiz-Frutos C, Delclós J, Ronda E, Garcia AM, Benavides F, editores. Salud Laboral, Conceptos y técnicas para la prevención de riesgos laborales. Barcelona: Elsevier Masson; 2014. p 27-36.

2. Benavides FG; Delclós J; Serra C. Estado de bienestar y salud pública: el papel de la salud laboral. Gac Sanit. 2018;32:377-80.

3. Real Decreto 39/1997, por el que se aprueba el Reglamento de los Servicios de Prevención. BOE núm. 27 de 17 Ene 1997.

4. Generalitat de Cataluña. Agència de Salut Pública de Cataluña. Unitats de Salut Laboral al territori [citado 10 Mar 2021]. Disponible en: https://salutpublica.gencat. cat/ca/ambits/salut_laboral/xarxa_d_unitats_de_salut_laboral/.

5. Ley 18/2009 de Salud Pública. BOE núm. 276, de 16 de noviembre de 2009.

6. Real Decreto 843/2011, por el que se establecen los criterios básicos sobre la organización de recursos para desarrollar la actividad sanitaria de los servicios de prevención. BOE núm. 158, de 04/07/2011.

7. Thangaratinam S, Redman CW. The Delphi technique. Obstet Gynaecol. 2005; $7: 120-5$.

8. McGraw KO, Wong SP. Forming inferences about some intraclass correlation coefficients. Psychological Methods. 1996;1:30-46.

9. van Vilsteren $\mathrm{M}$, van Oostrom $\mathrm{SH}$, de Vet HC, Franche RL, Boot $\mathrm{CR}$, et al. Workplace interventions to prevent work disability in workers on sick leave (Review). Cochrane Database Syst Rev. 2015;10: CD006955.

10. Vargas-Prada S, Demou E, Lalloo D, Avila-Palencia I, Sanati KA, et al. Effectiveness of very early workplace interventions to reduce sickness absence: A systematic review of the literature and meta-analysis. Scand $J$ Work Env Heal. 2016;42:261-72.

11. Nigatu YT, Liu Y, Uppal M, McKinney S, Rao S, et al. Interventions for enhancing return to work in individuals with a common mental illness: Systematic review and meta-analysis of randomized controlled trials. Psychol Med. 2016;46:3263-74.

12. Vaquero-Álvarez M, Álvarez-Theurer E, Salda R. Influencia de las condiciones de trabajo sobre la incapacidad temporal por contingencias comunes. Atención Primaria. 2018;50:238-46. 
13. Terradillos García MJ, Aguado Benedí MJ, García Puerto M, Saínz Gonzalez J, Cabello Herranz MJ, et al. Guía de Valoración de Incapacidad Laboral para Médicos de Atención Primaria (2a edición). Escuela Nacional de Medicina del Trabajo (ENMT). Instituto de Salud Carlos III. Ministerio de Economía y Competitividad; 2015.

14. Ramos RR. El Médico Inspector de la Seguridad Social. Med Segur Trab (Madr). 2014;1:12-6.

15. Rodríguez-Jareño MC, Molinero E, De Montserrat J, Valles A, Aymerich M. Calidad y adecuación técnica de la normativa de los exámenes de vigilancia de la salud dela población trabajadora en relación con su finalidad preventiva. Arch Prev Riesgos Labor. 2016;19:146-65.

16. Beach J, Watt D. General practitioners and occupational health professionals. BMJ. 2003;327:302-3.

17. Beyer S, Gouyet T, Letourneux VD, Mener E, Huger S, et al. Interprofessional Communication Concerning Work-Related Musculoskeletal Disorders: A Qualitative Study. J Occup Rehabil. 2018;28:721-9.

18. Persechino B, Fontana L, Buresti G, Rondinone BM, Laurano P, et al. Collaboration of occupational physicians with national health system and general practitioners in Italy. Ind Health. 2017:55:180-91.

19. Stratil JM, Rieger MA, Völter-Mahlknecht S. Cooperation between general practitioners, occupational health physicians, and rehabilitation physicians in Germany: what are problems and barriers to cooperation? A qualitative study. Int Arch Occup Environ Health. 2017;90:481-90.

20. Lydell M, Hildingh C, Söderbom A, Ziegert K. Future challenges for occupational health services can be prevented by proactive collaboration with the companies using the services: A participatory and reflection project. J Multidiscip Healthc. 2017;10:217-25.

21. Rodríguez-Jareño MC, Molinero E, de Montserrat J, Vallès A, Aymerich M. Do workers' health surveillance examinations fulfill their occupational preventive objective? Analysis of the medical practice of occupational physicians in Catalonia, Spain. Int J Occup Med Environ Health. 2017;30:823-48. 


\section{Tabla anexa 1:}

\section{Dificultades identificadas por los participantes de los SPP en la primera ronda}

1. Desconocimiento de la actividad sanitaria de los Servicios de prevención por parte de las personas trabajadoras y empresarias.

\footnotetext{
2. Poca valoración de la especialidad de medicina del trabajo.

3. Carencia de comunicación con el personal sanitario del Sistema público de salud.

4. Alto volumen de trabajo.

5. Tener que dedicar la mayor parte del tiempo a la realización de exámenes de salud.

6. La empresa pide a los especialistas de medicina del trabajo actividades no relacionadas con la prevención de riesgos laborales sino con la gestión de los recursos humanos.
}

7. Dificultades para gestionar las personas que son aptas con limitaciones (adaptación del puesto de trabajo, cambio de puesto de trabajo)

8. Dificultades relacionadas con los programas informáticos.

9. Hacer una vigilancia colectiva de la salud de poca calidad

10. Dispersión geográfica de los trabajadores y trabajadoras

11. Dificultades para poner en marcha protocolos de actuación consensuados por carencia de compromiso de la jerarquía de la empresa

12. Que el/la jefe del Servicio de prevención no sea uno/a profesional sanitario/aria

13. Volumen elevado de tareas administrativas

14. Evaluaciones de riesgos laborales no adecuadas para la aplicación de protocolos de vigilancia de la salud

15. Poco apoyo de los representantes sindicales.

16. Disparidad de criterios con las valoraciones del ICAM.

17. Carencia de tiempo para hacer estudios y publicaciones.

18. Dificultades para acceder a la información del SPA que realiza los reconocimientos médicos.

19. Dificultades para diferenciar la patología laboral de la no laboral.

20. Falta de participación en las decisiones sobre la compra de equipos de protección individual y equipos de trabajo

21. Elevado número de responsables intermedios que dificulta la gestión de la vigilancia de la salud.

22. Dificultades para hacer promoción de la salud.

23. Carencia de formación específica continuada adaptada a las necesidades del personal sanitario de los servicios de prevención.

24. Dificultades de relación con la Mutua

25. Dificultades para gestionar las situaciones de riesgo por embarazo

26. Heterogeneidad de puestos de trabajo que dificulta las actividades de vigilancia.

27. No tener la certeza que la persona trabajadora recibe el examen de salud enviado por vía telemática

28. Dificultades para hacer el seguimiento de los trabajadores y trabajadoras más allá del examen de salud.

29. No poder extender recetas médicas a los trabajadores y trabajadoras

30. Poca concordancia entre las directrices legales que tiene que seguir la vigilancia de la salud y la evidencia científica 
31. Poco espíritu de colaboración entre personal técnico de prevención y profesionales sanitarios

32. Poca claridad en la ley en cuanto a la obligatoriedad o no de los exámenes de salud.

33. Dificultades por parte de la propia empresa para aceptar la decisión del SPP en relación a si un trastorno es laboral o no.

34. Riesgo que la valoración de la aptitud médica derive en una herramienta de selección de personal.

35. El proceso de la determinación de contingencia es muy lento.

36. Carencia de ayuda por parte de la Administración pública.

37. Mandos que dan directrices poco claras que hace difícil la coordinación entre el personal técnico de prevención y el sanitario.

38. Poca reflexión por parte del personal especialista en medicina del trabajo respecto a lo que marcan las directrices legales, lo que se hace en realidad y lo que habría que hacer atendiendo a criterios profesionales.

39. Largos periodos de tiempos entre la propuesta de medidas y la implementación de estas.

40. Dificultad para obtener información válida respeto el estado de vacunación de los trabajadores y trabajadoras

41. No tener claro si los SPP deben intervenir en el control y seguimiento de la incapacidad temporal.

42. Las evaluaciones de riesgos psicosociales no se hacen o se hacen incompletas.

43. Confusión entre acoso y relaciones interpersonales conflictivas.

44. Falta de integración de la prevención dentro de la empresa

45. Falta de un sistema de información para la gestión de la prevención de riesgos laborales a la empresa

46. Dificultades para hacer llegar la información a todos los trabajadores y trabajadoras

47. Dificultades para establecer canales de comunicación y colaboración con las Unidades de salud laboral. 


\section{Tabla anexa 2:}

\section{Necesidades identificadas por los participantes de los SPP en la primera ronda.}

1. Crear grupos de trabajo multidisciplinarios (ICAM, Mutua, Unidad de salud laboral, SPP, SPA) para compartir experiencias y buscar soluciones

2. Establecer una vía de comunicación con el personal sanitario de los Centros de Atención Primaria.

3. Homogeneizar los criterios de control de la Inspección de Trabajo respeto los servicios de prevención de la administración pública y los de las empresas privadas.

\begin{tabular}{l}
\hline 4. Disponer de evaluaciones de riesgos específicas de los puestos de trabajo. \\
\hline 5. Establecer criterios claros de limitaciones/restricciones según la patología y el puesto de trabajo. \\
\hline 6. Disponer de programas que aborden el envejecimiento de la población. \\
\hline 7. Crear grupos de trabajo interdisciplinarios formados por SPP de diferentes empresas.
\end{tabular}

8. Disponer de un apoyo informático que permita la integración de toda la información interna útil para la vigilancia de la salud (historia médico-laboral, evaluaciones de riesgos, medidas preventivas, etc.)

9. Disponer de programas informáticos que faciliten la realización de estudios epidemiológicos.

10. Que la empresa ponga los medios y la voluntad para poner en marcha los protocolos de actuación.

11. Vías directas de relación entre el personal sanitario del servicio de prevención con el empresario/a o recursos humanos.

12. Disponer de recursos humanos suficientes.

13. Disponer de guías de buena praxis y protocolos de vigilancia de la salud basados en el consenso de los profesionales de la medicina del trabajo, adaptados a la realidad y con evidencia científica.

14. Implementar estrategias de reorganización del trabajo que permitan disponer de más tiempo para hacer investigación.

15. Mejorar la valoración de la prevención de riesgos laborales a la empresa con la implicación de la dirección de esta y el apoyo del Departamento de empresa y conocimiento.

16. En los SPP del ámbito sanitario, separar los circuitos asistenciales de los pacientes de los del servicio de prevención, para mantener la confidencialidad.

17. Formación en psiquiatría.

18. Que los trabajadores/as puedan consultar directamente con el médico/a del trabajo los problemas de salud que consideren relacionados con el trabajo

19. Modificar el circuito de determinación de la contingencia para evitar el impacto negativo en la salud de las personas trabajadoras (por ejemplo que tengan que hacer frente a las discrepancias entre el Servicio Público de Salud y la mutua o que los periodos de resolución sean muy largos)

\begin{tabular}{l}
\hline 20. Aumentar el apoyo administrativo. \\
21. Trabajo conjunto y mejora de la comunicación del equipo de medicina del trabajo y el personal \\
técnico de prevención. \\
\hline 22. Mayor integración de la prevención en las organizaciones sanitarias. \\
\hline 23. Dotar de equipos humanos y materiales estables para poder llevar a cabo los exámenes de salud \\
planificados.
\end{tabular}

24. Aumentar el recursos económicos tanto por parte de las empresas como de la administración pública.

25. Eliminar las aptitudes como herramienta de selección y orientarlas hacia las recomendaciones. 
26. Establecer un sistema de comunicación entre todo el personal sanitario implicado en la atención a los trabajadores y trabajadoras que permita tener acceso a toda la información médica con la máxima confidencialidad.

27. Realizar el control de la incapacidad temporal con normas legales claras.

28. Formación sanitaria continuada.

29. Formación en medicina evaluadora.

30. Poder aplicar medidas preventivas cuando sean necesarias sin tener que esperar largos periodos de tiempos debidos a la organización de la actividad de la propia empresa.

31. Realizar el seguimiento de toda la actividad sanitaria externa relacionada con la prevención de riesgos laborales, por ejemplo la relación con la mutua en los casos de accidente de trabajo.

32. Mejorar la formación de los mandos del personal en prevención de riesgos laborales para favorecer su colaboración.

33. Homogeneizar los criterios de solicitud de información a los SPP por parte de las administraciones autonómica y estatal.

34. Definir criterios para una adecuada vigilancia colectiva de la salud.

35. Que las sociedades científicas estimulen la participación y creación de grupos de trabajo con metodologías más ágiles y dinámicas para elaborar protocolos útiles para el día a día.

36. Disponer de diferentes indicadores de salud que permitan la planificación de la vigilancia de la salud.

37. Aumentar los recursos dedicados a la promoción de la salud.

38. Crear un clima de confianza para incentivar que las mujeres embarazadas comuniquen su estado lo antes posible.

39. Formación en estadística y epidemiología

40. Hacer las evaluaciones de riesgos conjuntamente con el personal técnico sanitario y el no sanitario.

41. Dar a conocer las funciones, competencias y utilidad de la actividad sanitaria de los SPRL a diferentes agentes: sociedad, médicos de familia, otros profesionales, etc.

42. Establecer colaboración con los médicos del ICAM.

43. Participar en la planificación e implementación de la formación en prevención de riesgos laborales a los trabajadores y trabajadoras

44. Crear diálogo y relación de confianza y colaboración entre empresa y mutua para gestionar las situaciones de embarazo

45. Crear grupos de trabajo con profesionales de otras especialidades para mejorar la detección y gestión de las enfermedades profesionales.

46. Valorar la posibilidad de hacer recetas en la asistencia de urgencia.

47. Conocer el estado de vacunación del personal en aquellos casos en que sea necesario por su exposición.

48. Mejorar los sistemas de información en salud laboral.

49. Informar a los trabajadores y trabajadoras que el personal sanitario de los SPP no hacen recetas médicas. 


\section{Tabla anexa 3:}

\section{Dificultades identificadas por los participantes de los SPA en la primera ronda.}

1. Dificultades para conocer a fondo los puestos de trabajo porque las evaluaciones de los lugares de trabajo son poco precisas.

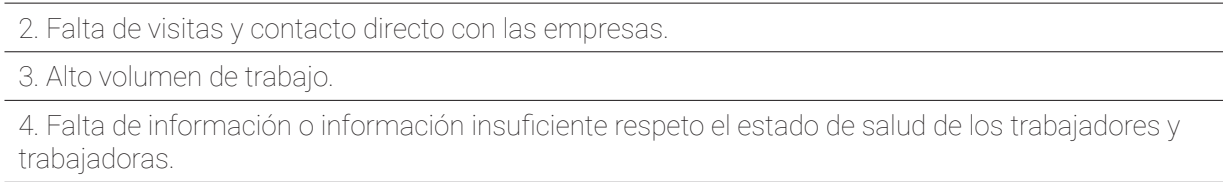

5. Falta de conocimiento de la medicina del trabajo por parte de la persona responsable del servicio de prevención.

6. Desconocimiento de la actividad sanitaria de los servicios de prevención por parte de las personas trabajadoras y empresarias.

7. Presión de las empresas para obtener resultados de acuerdo con sus intereses.

8. Falta de comunicación entre el personal sanitario implicado en la salud de los trabajadores y trabajadoras (Servicio de prevención, médico/a de familia, especialista, Mutua, Unidad de salud laboral, ...)

9. Dificultades para hacer la vigilancia colectiva de la salud (por ejemplo falta de tiempo, programas informáticos inadecuados).

10. Que prevalezcan criterios económicos por sobre los de calidad.

11. Presión de tiempo por parte de las empresas clientes.

12. Tener que dedicar la mayor parte del tiempo a la realización de exámenes de salud.

13. Imposibilidad de solicitar pruebas complementarias

14. Demanda por parte de los trabajadores y trabajadoras de pruebas no específicas para sus riesgos.

15. Carencia de homogeneidad de criterios en algunas de las actividades del servicio de prevención, por ejemplo: periodicidad de los exámenes de salud, criterios de aptitudes.

16. Carencia de evaluaciones de riesgos laborales.

17. Dificultades para hacer promoción de la salud.

18. Falta de comunicación con el personal sanitario del Sistema público de salud.

19. Realización de exámenes de salud por médicos y médicas que no son especialistas en medicina del trabajo.

20. Falta de claridad de la legislación sobre condiciones mínimas por los reconocimientos médicos en caso de desplazamiento a las empresas.

21. Actitud de los empresarios que contratan un SPA y creen que no sirve para nada, lo hacen por obligación legal.

22. Negativa de los trabajadores y trabajadoras a hacer los exámenes de salud

23. Coste elevado de algunas pruebas en caso de exposición a algunos riesgos concretos (biológicos, radiaciones, amianto, etc.).

24. Poca variedad en los casos que se atienden.

25. Dificultades para hacer el seguimiento de los trabajadores y trabajadoras más allá del examen de salud.

26. Falta de formación específica continuada adaptada a las necesidades del personal sanitario de los servicios de prevención. 
27. Pérdida de seguimiento de los trabajadores y trabajadoras que se derivan a la Mutua por sospecha de patología profesional

28. Falta de comunicación con las mutuas.

29. Dificultades de comunicación con los mandos superiores.

30. Tener que hacer exámenes de salud en unidades móviles

31. Dificultades para hacer la declaración de enfermedades profesionales

32. Falta de comunicación entre el personal sanitario de las diferentes delegaciones del propio SPA.

33. Dificultades para hacer investigación.

34. Dificultades para participar en actividades como jornadas, congresos, encuentros formativos

35. Baja remuneración económica.

36. Cuestionamiento del criterio médico por parte de las empresas en relación a la valoración de la aptitud.

37. Disparidad de criterios con las valoraciones del ICAM (Instituto Catalán de Evaluaciones Médicas)

38. Programación de los exámenes de salud sin tener en cuenta los descansos de los profesionales sanitarios del SPA.

39. Las persones empresarias limitan las actividades sanitarias del Servicio de prevención por el coste económico

40. Falta de actualización de los equipos de los SPA

41. No disponer de vacunas.

42. Poca valoración de la especialidad de medicina del trabajo.

43. Consideración de los exámenes de salud como un trámite administrativo por parte de los trabajadores y trabajadoras

44. Algunas personas trabajadoras no entienden la necesidad de cuidar su salud.

45. Dificultades para impartir formación a los trabajadores y trabajadoras

46. Carencia de representación de la medicina del trabajo en los equipos directivos de los servicios de prevención.

47. No poder realizar tareas de primeros auxilios por no estar presencialmente en las empresas.

48. Falta de comunicación entre el personal sanitario de los diferentes servicios de prevención.

49. Hacer una vigilancia colectiva de la salud de poca calidad 


\section{Tabla anexa 4:}

\section{Necesidades identificadas por los participantes de los SPA en la primea ronda.}

1. Conocer mejor las empresas clientes.

2. Participar en la realización de la evaluación de riesgos

3. Establecer un sistema de comunicación entre todo el personal sanitario implicado en la atención a los trabajadores y trabajadoras que permita tener acceso a toda la información médica con la máxima confidencialidad.

\begin{tabular}{l}
\hline 4. Formación sanitaria continuada. \\
\hline 5. Aumentar la influencia de los médicos y médicas de medicina del trabajo en las empresas. \\
6. Implementar algún sistema para que el coste de las pruebas específicas no recaiga únicamente \\
en el empresario. \\
\hline 7. Formación en promoción de la salud. \\
\hline 8. Disminuir el ritmo de trabajo. \\
\hline 9. Disminuir el número de reconocimientos médicos que se tienen que realizar. \\
\hline 10. Aumentar el número de especialistas de medicina del trabajo. \\
\hline 11. Tener más tiempo para realizar las visitas. \\
12. Disponer de evaluaciones de riesgos específicas de los puestos de trabajo.
\end{tabular}

13. Reuniones periódicas con el ICAM.

14. Aumentar la presencia de profesionales de la medicina del trabajo en los lugares directivos de los SPA.

15. Control y apoyo por parte de la Administración.

16. Trabajo conjunto y mejora de la comunicación del equipo de medicina del trabajo y el persona técnico de prevención

17. Cambiar el modelo de organización de los SPA, de forma que dependan de organismos públicos con criterios y precios comunes.

18. Incrementar el reconocimiento de la medicina del trabajo por parte de empresas y personas trabajadoras

19. Informar, formar y sensibilizar las persones empresarias y trabajadoras sobre la importancia de la vigilancia de la salud

20. Mejorar la remuneración de los profesionales sanitarios

21. Tener tiempo para hacer otras tareas de la medicina del trabajo que no sean la vigilancia de la salud únicamente.

22. Mejorar el control de los SPA en relación a las ratios que determina la normativa vigente

23. Tener tiempo suficiente para realizar visitas a las empresas.

24. Que haya más oferta de formación, que sea más económica y que haya más en formato en línea.

25. Establecer un sistema en que el médico o médica pueda denunciar abusos por parte de las empresas, sin tener miedo a perder su trabajo.

26. Promulgar leyes que impiden los abusos de las empresas en relación a la vigilancia de la salud.

27. Que la actuación de la Administración se base en premiar las buenas prácticas, más que al sancionar las actuaciones incorrectas.

28. Eliminar la subcontratación de centros no acreditados para hacer vigilancia de la salud

29. Poder realizar interconsultas a especialistas. 
30. Mejorar la relación del personal médico del SPA con el de atención primaria.

31. Realizar encuentros entre profesionales de salud laboral de la misma zona.

32. Prohibición por ley de hacer exámenes de salud laboral en unidades móviles.

33. Disponer de programas informáticos que faciliten la realización de estudios epidemiológicos

34. Aumentar la formación de los MIR y mejorar su residencia consiguiendo más implicación por parte de las mutuas, SPA y hospitales

35. Que la Unidad de Salud Laboral local haga de puente con el Sistema Público de Salud y las mutuas

36. Más interés y compromiso por parte de las empresas

37. Actualizar los equipos de los SPA.

38. Dar estímulos positivos a la comunicación de enfermedades profesionales, facilitando y premiando su investigación y corrección de causas,

39. Aumentar el precio del examen de salud para incrementar la rentabilidad.

40. Que las autoridades laborales no identifiquen la vigilancia de la salud con revisiones y certificado de aptitud.

41. Definir criterios para una adecuada vigilancia colectiva de la salud.

42. Que las empresas dejen entrar a trabajar a los trabajadores y trabajadoras con un apto con limitaciones.

43. Facilitar que los médicos y médicas del trabajo puedan estudiar las sospechas de enfermedad profesional, participar en el proceso curativo y la valoración antes de la reincorporación.

44. Establecer por ley unos precios mínimos de las actividades sanitarias de los SPA.

45. Crear más espacios de conexión entre los profesionales del sector

46. Establecer a la agenda de visitas un descanso obligatorio

47. Facilitar la asistencia y participación a actas de difusión de la especialidad, por ejemplo modificando la normativa o a través de patrocinios y ayudas

48. Establecer criterios claros de uso de unidades móviles y hacer un control.

49. Mejorar los informes de vigilancia de la salud colectiva con herramientas específicas y con valor estadístico

50. Establecer claramente las competencias de cada profesional del SPA con criterios objetivos y verificables

51. Valorar el funcionamiento de los servicios de prevención de acuerdo con la evidencia científica

52. Mejorar los protocolos de vigilancia de la salud según la medicina basada en la evidencia

53. Disminuir el número de empresas a las que se tiene que dar servicio

54. Poder realizar todas las actividades de la medicina del trabajo y no únicamente la vigilancia de la salud.

55. Establecer una legislación de prevención de riesgos laborales más concreta y específica que apueste para un mayor contenido en medicina del trabajo y no solo el hacer reconocimientos médicos.

56. Incrementar el control de la Inspección de trabajo sobre las tareas de vigilancia de la salud. 